\title{
Bimeromorphic geometry of Kähler threefolds
}

\author{
Andreas Höring and Thomas Peternell
}

\begin{abstract}
We describe the recently established minimal model program for (non-algebraic) Kähler threefolds as well as the abundance theorem for these spaces.
\end{abstract}

\section{Introduction}

Given a complex projective manifold $X$, the Minimal Model Program (MMP) predicts that either $X$ is covered by rational curves ( $X$ is uniruled) or $X$ has a - slightly singular - birational minimal model $X^{\prime}$ whose canonical divisor $K_{X^{\prime}}$ is nef; and then the abundance conjecture says that some multiple $m K_{X^{\prime}}$ is spanned by global sections (so $X^{\prime}$ is a good minimal model). The MMP also predicts how to achieve the birational model, namely by a sequence of divisorial contractions and flips. In dimension three, the MMP is completely established (cf. Kwc92, KM98 for surveys), in dimension four, the existence of minimal models is established (BCHM10, Fuj04, Fuj05]), but abundance is wide open. In higher dimensions minimal models exists if $X$ is of general type [BCHM10; abundance not being an issue in this case.

In this article we discuss the following natural

QUESTION 1.1. Does the MMP work for general (non-algebraic) compact Kähler manifolds?

Although the basic methods used in minimal model theory all fail in the Kähler case, there is no apparent reason why the MMP should not hold in the Kähler category. And in fact, in recent papers [HP16], HP15] and [CHP16, the Kähler MMP was established in dimension three:

THEOREM 1.2. Let $X$ be a normal $\mathbb{Q}$-factorial compact Kähler threefold with terminal singularities. Then there exists a MMP (i.e. a finite sequence of divisorial contractions and flips)

$$
X \rightarrow X^{\prime}
$$

such that the following holds:

- If $X$ is not covered by rational curves, then $X^{\prime}$ is a good minimal model: there exists a $m \in \mathbb{N}$ such that $m K_{X^{\prime}}$ is generated by its global sections.

2010 Mathematics Subject Classification. Primary 32J27, 14E30, 14J35, 14J40, 14M22, $32 \mathrm{~J} 25$. 
- If $X$ is covered by rational curves, then $X^{\prime}$ is a Mori fibre space: there exists a fibration $\varphi: X^{\prime} \rightarrow Y$ such that $-K_{X^{\prime}}$ is $\varphi$-ample and $b_{2}\left(X^{\prime}\right)=$ $b_{2}(Y)+1$.

Two main issues in the MMP are to construct "contractions of extremal rays" and to construct $K_{X}$-negative rational curves. In the algebraic case, contractions are constructed by exhibiting nef line bundles of the form $K_{X}+H$ with $H$ ample. Suitable multiples of these line bundles are spanned and then the contraction is given by the sections of the line bundle. Rational curves are constructed using reduction to char $p$. Both methods fundamentally fail in the Kähler setting. One reason is of course, that there are only a few line bundles (and no ample line bundles) on a general Kähler manifold, and also the Mori cone $\overline{\mathrm{NE}}(X)$ is too small to be relevant. At least in dimension three, it is nevertheless possible to construct good minimal models. The aim of this article is to explain how this is achieved, including the necessary framework of Kähler geometry. To give a flavor of the difficulties arising in the Kähler setting, we mention the following

Conjecture 1.3. Let $X$ be a compact Kähler manifold such that the canonical class is not nef (i.e, the first Chern class $c_{1}\left(K_{X}\right)$ is not in the closure of the Kähler cone). Then there exists a rational curve $C$ such that $K_{X} \cdot C<0$.

As we will see in the next section it is even not clear whether there is any curve $C$ such that $K_{X} \cdot C<0$ !

\section{Brief review of the algebraic case}

Let $X$ be a complex projective variety, and let $\mathrm{NS}(X)$ be its Néron-Severi group. We denote by $N^{1}(X):=\mathrm{NS}(X) \otimes \mathbb{R}$ the corresponding real vector space, and by $N_{1}(X)$ its dual, the vector space generated by classes of curves. By definition a class $\alpha \in N^{1}(X)$ is nef if it is in the closure of the cone generated by ample divisors. This somewhat abstract definition (which has the advantage of generalising to the Kähler case, cf. Section 3) can be translate in more geometric terms by Kleiman's criterion. We have

$$
\alpha \in N^{1}(X) \text { is nef } \Leftrightarrow \alpha \cdot C \geq 0 \quad \forall C \subset X \text { a curve }
$$

One of the cornerstones of the minimal model program is to give a much more precise description of nefness when $\alpha$ is the class defined by the canonical divisor; for all definitions and basic results around the MMP we refer e.g. to KMM87. and KM98.

THEOREM 2.1. Let $X$ be a normal $\mathbb{Q}$-factorial projective variety with terminal singularities. Then there exists an at most countable family $\left(\Gamma_{i}\right)_{i \in I}$ of rational curves on $X$ such that

$$
0<-K_{X} \cdot \Gamma_{i} \leq 2 \operatorname{dim} X-1
$$

and

$$
\overline{N E}(X)=\overline{N E}(X)_{K_{X} \geq 0}+\sum_{i \in I} \mathbb{R}^{+}\left[\Gamma_{i}\right] .
$$

Given a projective variety $X$, the aim of the minimal model program is to replace $X$ by some birational model such that either the canonical divisor is nef or we have a Mori fibre space structure. The way to get closer to this model is to contract the extremal rays appearing in the cone theorem. The existence of these contractions is assured by the contraction theorem: 
TheOREM 2.2. Let $X$ be a normal $\mathbb{Q}$-factorial projective variety with terminal singularities. Let $R$ be a $K_{X}$-negative extremal ray in $\overline{N E}(X)$. Then there exists a morphism with connected fibres $\varphi: X \rightarrow Y$ onto a normal projective variety $Y$ such that a curve $C \subset X$ is contracted onto a point if and only if $[C] \in R$.

We call $\varphi$ the elementary Mori contraction associated to the extremal ray $R$.

The statements of Theorem 2.1 and Theorem 2.2 make sense in the more general setting of compact Kähler spaces, and one expects them to hold in arbitrary dimension. However we will see in Section 4 that even for threefolds this requires a substantial amount of work. The first reason is that Kleiman's theorem (2.1) is not true for arbitrary classes on non-algebraic Kähler manifolds, but in order for the MMP to exist it should hold for the canonical class. The second reason is hidden in the proof of Theorem 2.2 a morphism $\varphi: X \rightarrow Y$ between projective varieties is always defined by some globally generated line bundle, so the natural way to prove Theorem 2.2 is to give sufficient conditions for a line bundle to be semiample. This is achieved by the basepoint free theorem:

TheOREM 2.3. Let $X$ be a normal $\mathbb{Q}$-factorial projective variety with terminal singularities. Let $L$ be a nef line bundle such that $L-K_{X}$ is nef and big. Then $m L$ is generated by its global sections for all $m \gg 0$.

This very important technical result will not be of any help if we want to consider general Kähler spaces: the existence of a line bundle $L-K_{X}$ that is nef and big implies that $X$ is Moishezon. Yet Moishezon spaces (with rational singularities) that are Kähler are always projective [Nam02. Thus an important part of the work will be to find new ways to prove the contraction theorem.

While the cone and contraction theorem need completely new proofs in the Kähler setting, other parts of the minimal model program can be easily generalised from the projective case: since we always contract $K_{X}$-negative extremal rays, the contractions are projective morphisms polarised by $-K_{X}$. Moreover the existence of flips in dimension three was proven by Mori Mor88 and Shokurov Sho92 in the setting of complex spaces, so we can use directly their results.

The notion of $\mathbb{Q}$-factoriality is important to run the MMP. For a non-projective complex space the canonical sheaf might not be a $\mathbb{Q}$-divisor, so this requires a little care:

Definition 2.4. Let $X$ be a normal compact complex space. We say that $X$ is $\mathbb{Q}$-factorial, if every Weil divisor is $\mathbb{Q}$-Cartier and there is a number $m \in \mathbb{N}$ such that the coherent sheaf $\mathcal{O}_{X}\left(m K_{X}\right)=\left(\omega_{X}^{\otimes m}\right)^{* *}$ is locally free.

\section{Kähler spaces and the generalised Mori cone $\overline{\mathbf{N A}}(X)$}

In this section we introduce the cone $\overline{N A}(X)$ on a compact Kähler space $X$ which plays the role of the Mori cone $\overline{N E}(X)$ in the algebraic setting. The notion of a singular Kähler space was first introduced by Grauert Gra62].

Definition 3.1. An irreducible and reduced complex space $X$ is Kähler if there exists a Kähler form $\omega$, i.e. a positive closed real $(1,1)$-form $\omega \in \mathcal{A}_{\mathbb{R}}^{1,1}(X)$ such that the following holds: for every point $x \in X_{\text {sing }}$ there exists an open neighbourhood $x \in U \subset X$ and a closed embedding $i_{U}: U \subset V$ into an open set $V \subset \mathbb{C}^{N}$, and a strictly plurisubharmonic $C^{\infty}$-function $f: V \rightarrow \mathbb{C}$ with $\left.\omega\right|_{U \cap X_{\text {nons }}}=$ $\left.(i \partial \bar{\partial} f)\right|_{U \cap X_{\text {nons }}}$. 
For the notion and basic properties of $(p, q)$-forms and currents on singular spaces we refer to Dem85. We will denote by $\mathcal{A}_{X}^{p, q}$ the sheaf of $(p, q)$-forms, and by $\mathcal{D}_{X}^{p, q}$ the sheaf of currents of bidegree $(p, q)$. The relevant cohomology theory on Kähler spaces is the Bott-Chern cohomology, see [BPEG13, Defn. 4.6.2]:

Definition 3.2. Let $X$ be a normal complex space. Let $\mathcal{H}_{X}$ be the sheaf of real parts of holomorphic functions multiplied with $i$. A (1,1)-form (resp. (1,1)current) with local potentials on $X$ is a global section of the quotient sheaf $\mathcal{A}_{X}^{0,0} / \mathcal{H}_{X}$ (resp. $\left.\mathcal{D}_{X}^{0,0} / \mathcal{H}_{X}\right)$. Then the Bott-Chern cohomology is defined as

$$
H_{\mathrm{BC}}^{1,1}(X):=H^{1}\left(X, \mathcal{H}_{X}\right)
$$

REMARK 3.3. An element of the Bott-Chern cohomology group can be viewed as a closed $(1,1)$-form with local potentials modulo all the forms that are globally of the form $d d^{c} u$. Alternatively, we can also use $(1,1)$-currents with local potentials to define Bott-Chern cohomology.

To make the analogy to the projective case clearer, we define

Definition 3.4. Let $X$ be a normal compact complex space in the Fujiki class C. Then

$$
N^{1}(X):=H_{\mathrm{BC}}^{1,1}(X) .
$$

The algebraic definition deals with classes of divisors; however in the nonalgebraic setting there are too few divisors, so that this space is too small to be useful. If $X$ has rational singularities - which will always be the case in our considerations - then

$$
N^{1}(X) \subset H^{2}(X, \mathbb{R}) .
$$

Even in the algebraic case, the new space $N^{1}(X)$ can be larger than the traditional space $N^{1}(X)=N S(X) \otimes \mathbb{R}$.

If $X$ is projective, then the space $N_{1}(X)$ is the subspace of $H_{2}(X, \mathbb{R})$ generated by classes of curves. Again we need a more general definition here.

Definition 3.5. Let $X$ be a normal compact complex space in class $\mathcal{C}$. We define $N_{1}(X)$ to be the vector space of real closed currents of bidimension $(1,1)$ modulo the following equivalence relation: $T_{1} \equiv T_{2}$ if and only if

$$
T_{1}(\eta)=T_{2}(\eta)
$$

for all real closed $(1,1)$-forms $\eta$ with local potentials.

In this setting, the analytic counterpart of the Mori cone $\overline{N E}(X)$ in the projective case is given by

Definition 3.6. Let $X$ be a normal compact complex space in class $\mathcal{C}$. Then $\overline{\mathrm{NA}}(X) \subset N_{1}(X)$ is the closed cone generated by the classes of positive closed currents. The Mori cone is the closed subcone

$$
\overline{\mathrm{NE}}(X) \subset \overline{\mathrm{NA}}(X)
$$

generated by those positive closed currents arising as currents of integration over curves.

If $X$ is projective, $\overline{\mathrm{NE}}(X)$ is just the usual Mori cone of curves. However, even if $X$ is a projective manifold, the cone $\overline{\mathrm{NA}}(X)$ can be larger than $\overline{\mathrm{NE}}(X)$, namely when $\rho(X)<h^{1,1}(X)$. 
Definition 3.7. Let $X$ be a normal compact complex space and $u \in H_{\mathrm{BC}}^{1,1}(X)$.

(1) $u$ is pseudo-effective, if $u$ can be represented by a current $T \in \mathcal{D}^{1,1}(X)$ which is locally of the form $\partial \bar{\partial} \varphi$ with a plurisubharmonic function $\varphi$.

(2) Then $u$ is nef if $u$ can be represented by a form $\alpha$ with local potentials such that for some positive $(1,1)-$ form $\omega$ on $X$ and for every $\epsilon>0$ there exists a $C^{\infty}$-function $f_{\epsilon}$ such that

$$
\alpha+i \partial \bar{\partial} f_{\epsilon} \geq-\epsilon \omega .
$$

(3) $\operatorname{Nef}(X) \subset N^{1}(X)$ is the cone generated by nef cohomology classes.

The notion of nef divisors/classes is central for the MMP, so let us explain what the slightly technical definition above means. For a Kähler space nef classes are limits of positive (i.e. Kähler) classes:

Theorem 3.8. Dem92, Prop.6.1.iii)] Let $X$ be a normal compact Kähler space. Then $\operatorname{Nef}(X)$ is the closure of the Kähler cone

More geometrically we know that a $\mathbb{Q}$-Cartier divisor $u$ on a normal projective variety $X$ is nef if $u \cdot C \geq 0$ for all irreducible curves $C \subset X$. On a non-algebraic Kähler space such a divisor can even be antinef! Indeed if $X$ is a compact Kähler surface of algebraic dimension one, then we have an elliptic fibration $\varphi: X \rightarrow B$ onto a projective curve $B$. Set $u:=-\varphi^{*} H$ with $H$ an ample divisor on $B$, so $u$ is antinef. However all the curves $C \subset X$ are contracted by $\varphi$, so we have $u \cdot C=0$ for all curves $C \subset X$. We will explain in Section 4 that this phenomena should not happen when $u=c_{1}\left(K_{X}\right)$.

In the projective setting, the nef cone (i.e., the closure of the ample cone) and the Mori cone $\overline{\mathrm{NE}}(X)$ are dual. Here is the analogue in the Kähler case: given a real closed $(1,1)$-form $\omega$ on $X$ with local potentials, we define

$$
\lambda_{\omega} \in N_{1}(X)^{*},[T] \mapsto T(\omega) .
$$

Notice that if $T(\omega)=0$ for all closed currents $T$ of bidimension $(1,1)$, then $\lambda_{\omega}=0$. Thus we obtain well-defined canonical map

$$
\Phi: N^{1}(X) \rightarrow N_{1}(X)^{*},[\omega] \mapsto \lambda_{\omega} .
$$

TheOREM 3.9. Let $X$ be a normal compact complex space in class $\mathcal{C}$ with only rational singularities.

(1) $\Phi$ is an isomorphism.

(2) If $\operatorname{dim} X=3$, then $\Phi(\operatorname{Nef}(X))=\overline{N A}(X)$.

The assumption on the dimension in the last statement should be superfluous. Since the MMP is an iteration of morphisms and flips one has to be able to check that at every step we remain in the category of Kähler spaces. Here is an example of such a criterion:

Lemma 3.10. Let $X$ be a normal $\mathbb{Q}$-factorial compact Kähler threefold, and let $\varphi: X \rightarrow X^{\prime}$ be a bimeromorphic Mori contraction. Let $\alpha$ be a nef class on $X$ such that the following holds: $\alpha^{3}>0, \alpha^{2} \cdot S=0$ if and only if the surface $S$ is contracted by $\varphi$ and $\alpha \cdot C=0$ if and only if the curve $C$ is contracted by $\varphi$. Then $\alpha=\varphi^{*} \alpha^{\prime}$ with $\alpha^{\prime}$ a Kähler class on $X^{\prime}$. In particular $X^{\prime}$ is Kähler. 
Proof. It is easy to see that $\alpha=\varphi^{*} \alpha^{\prime}$ with $\alpha^{\prime} \in N^{1}\left(X^{\prime}\right)$ (cf. [HP16. Sect.3]), the interesting part is to show the Kähler property: since $\alpha^{3}>0$ the class $\alpha$ is nef and big [DP04] and as a consequence of [CT15, Bou04, applied on a resolution of singularities, we can choose a Kähler current $T$ in $\alpha$ such that its Lelong level sets are in the $\varphi$-exceptional locus. The push-forward $\varphi_{*}(T)$ defines a Kähler current $T^{\prime}$ in $\alpha^{\prime}$ such that its Lelong level sets are in the image of the $\varphi$-exceptional locus. By [DP04, Prop.3.3], see also [CT16, we are done if we show that the restriction of $\alpha^{\prime}$ to all these sets is a Kähler class. Yet $X$ has dimension three, so the image of the exceptional locus is either a union of points or a curve $C^{\prime} \subset X^{\prime}$. Now observe the following: the map $\varphi^{-1}\left(C^{\prime}\right) \rightarrow C^{\prime}$ is a projective morphism over a projective variety, so it has a multisection $C$. By the projection formula $\alpha^{\prime} \cdot C^{\prime}$ is a positive multiple of $\alpha \cdot C$ which (by our assumption on intersections) is positive. Thus $\left.\alpha^{\prime}\right|_{C^{\prime}}$ is a Kähler class.

REMARK 3.11. In HP16 the Kähler property was shown by a more general theorem [HP16, Thm.3.18] involving only conditions on $\overline{\mathrm{NA}}(X)$. S.Boucksom pointed out that for the fact in Step 2 of the proof that $T_{\infty}(E)<0$, some additional arguments should be given. We know in the situation of Step 2 of Theorem 3.18 that $\left[\mu_{*}\left(T_{\infty}\right)\right]=0$. Now we may write

$$
\left[T_{\infty}\right]=\left[i_{*}(R)\right]
$$

with a current $R$ supported on the exceptional divisor $E$. This is easily seen since there is a class $b \in H_{2}(E)$ such that $i_{*}(b)$ equals the class of $\left[T_{\infty}\right]$ in $H_{2}(\hat{X})$. Decompose $R=R^{+}-R^{-}$with positive closed currents $R^{+}$and $R^{-}$and define a positive closed $\hat{T}=T_{\infty}+i_{*}\left(R^{-}\right)$. Notice $\mu_{*}\left(i_{*}\left(R^{+}\right)\right)=0$ and let $\pi: \tilde{X} \rightarrow \hat{X}$ be a sequence of blow-ups such that $\tilde{X}$ is a compact Kähler manifold. Let $\tilde{S} \subset \tilde{X}$ and $\hat{S} \subset \hat{X}$ be the exceptional loci. Now Step 1 of 3.18 is easily adapted to yield

$$
\chi_{\hat{X} \backslash \hat{S}} \hat{T}=0 .
$$

Consequently, $T_{\infty}$ is supported on $\hat{S}$, hence $T_{\infty}(E)<0$.

\section{MMP for Kähler threefolds}

4.1. Existence of rational curves, bend-and-break. In this whole section we denote by $X$ a $\mathbb{Q}$-factorial compact Kähler threefold with terminal singularities, for the outlines of the proofs we will assume implicitly that $X$ is smooth. We also suppose:

The canonical class $K_{X}$ is not nef.

One of the first fundamental contributions of Mori was to translate this numerical property into a geometric statement:

Theorem 4.1. Mor79, Mor82 Let $X$ be a projective manifold such that $K_{X}$ is not nef. Then there exists a rational curve $C \subset X$ such that $-K_{X} \cdot C>0$.

Mori's proof uses deformation theory of curves and a reduction to positive characteristic in an essential way, and for a long time it was not clear how to generalise his statement in a non-algebraic setting. The approach used in [HP16, HP15 is to split the problem in two parts. The first part is due to M. Brunella: 
Theorem 4.2. Bru06 Let $X$ be a $\mathbb{Q}$-factorial compact Kähler threefold with terminal singularities. Then $K_{X}$ is not pseudoeffective if and only if $X$ is covered by rational curves.

First observe that we may assume $X$ to be smooth: if $\hat{X} \rightarrow X$ is any desingularisation, then $K_{\hat{X}}$ is not pseudoeffective if and only if $K_{X}$ is not pseudoeffective, the singularities of $X$ being terminal. Now Brunella proves that the canonical bundle $K_{\mathcal{F}}$ of a rank one foliation $\mathcal{F} \subset T_{X}$ is not pseudoeffective if and only if the general leaf of $\mathcal{F}$ is a rational curve. Since a non-algebraic Kähler threefold always admits a rank one foliation defined by a holomorphic two-form (by Kodaira's theorem), this implies the statement. For the discussion of non-uniruled spaces we may therefore replace (4.1) by

The canonical class $K_{X}$ is pseudoeffective, but not nef.

By the theorem of Demailly-Păun DP04 the existence of a cohomology class that is pseudoeffective, but not nef yields some first geometric information: there exists a proper subvariety $Z \subsetneq X$ such that $\left.K_{X}\right|_{Z}$ is not pseudoeffective. If $X$ is smooth (or at least Gorenstein 1 ) we can focus on the case where $Z$ is a surface (hence a divisor in the threefold $X$ ). Indeed if $Z$ is a curve $C$, then $\left.K_{X}\right|_{C}$ being not pseudoeffective is equivalent to $K_{X} \cdot C<0$. The deformation theory of curves on threefolds now shows that the deformations of $C$ cover a surface $S \subset X$ [Kol96, II, Thm.1.15]. Then the restriction $\left.K_{X}\right|_{S}$ is not pseudoeffective since $S$ is covered by $K_{X}$-negative curves.

Lemma 4.3. HP16, Lemma 4.1] Let $X$ be a $\mathbb{Q}$-factorial compact Kähler threefold with terminal singularities that is not uniruled. Let $S \subset X$ be an irreducible surface such that $\left.K_{X}\right|_{S}$ is not pseudoeffective. Then $S$ is covered by rational curves.

Proof. Since $K_{X}$ is pseudoeffective we can use Boucksom's decomposition theorem [Bou04]: there exist irreducible surfaces $S_{j} \subset X$ such that

$$
K_{X}=\sum_{j=1}^{r} \lambda_{j} S_{j}+N\left(K_{X}\right)
$$

where $\lambda_{j} \geq 0$ and the cohomology class $N\left(K_{X}\right)$ is pseudoeffective and the restriction of $N\left(K_{X}\right)$ to any surface in $X$ is pseudoeffective. One easily deduces that $S$ is one of the surfaces $S_{j}$, so up to renumbering we can write

$$
S=S_{1}=\frac{1}{\lambda_{1}} K_{X}-\frac{1}{\lambda_{1}}\left(\sum_{j=2}^{r} \lambda_{j} S_{j}+N\left(K_{X}\right)\right) .
$$

Yet by adjunction this implies

$$
K_{S}=\left.\left(K_{X}+S\right)\right|_{S}=\left.\frac{\lambda_{1}+1}{\lambda_{1}} K_{X}\right|_{S}-\frac{1}{\lambda_{1}}\left(\sum_{j=2}^{r} \lambda_{j}\left(S_{j} \cap S\right)+\left.N\left(K_{X}\right)\right|_{S}\right) .
$$

By hypothesis $\left.K_{X}\right|_{S}$ is not pseudoeffective and $-\frac{1}{\lambda_{1}}\left(\sum_{j=2}^{r} \lambda_{j}\left(S_{j} \cap S\right)+\left.N\left(K_{X}\right)\right|_{S}\right)$ is anti-pseudoeffective. Thus $K_{S}$ is not pseudoeffective, hence $S$ is covered by rational curves (pass to a desingularisation whose canonical class is still not pseudoeffective).

\footnotetext{
${ }^{1}$ For simplicity of the exposition, we completely ignore the substantial difficulties in the non-Gorenstein case, cf. [HP16, Sect.4.C, Sect.5].
} 
Put together these arguments show the existence of $K_{X}$-negative rational curves if $K_{X}$ is not nef. The difference between this existence result and the classical cone Theorem 2.1 is that we have to show that if for some curve $C \subset X$ the degree $-K_{X} \cdot C$ is too large (say $-K_{X} \cdot C>2 \operatorname{dim} X-1$ ), then the cohomology class $C$ decomposes into $[C]=\left[C_{1}\right]+\left[C_{2}\right]$ with $C_{i}$ effective 1-cycles. Mori proves this property via his famous bend-and-break technique, again a reduction to positive characteristic plays an important role. In our case we can use Kol96. Thm. 1.15] and reduce many arguments to considerations in one of the surfaces $S_{j}$ appearing in the decomposition (4.3).

Proposition 4.4. HP16, Thm.6.2] Let $X$ be a $\mathbb{Q}$-factorial compact Kähler threefold with terminal singularities that is not uniruled. Then there exists a countable family $\left(\Gamma_{i}\right)_{i \in I}$ of rational curves on $X$ such that

$$
0<-K_{X} \cdot \Gamma_{i} \leq 4
$$

and

$$
\overline{\mathrm{NE}}(X)=\overline{\mathrm{NE}}(X)_{K_{X} \geq 0}+\sum_{i \in I} \mathbb{R}^{+}\left[\Gamma_{i}\right]
$$

However, as observed in Section 3 this is not the statement that we want: the bimeromorphic geometry of Kähler manifolds is governed by $\overline{\mathrm{NA}}(X)$, not the much smaller $\overline{\mathrm{NE}}(X)$. Somewhat surprisingly, we have a precise analogue for $\overline{\mathrm{NA}}(X)$ :

TheOREM 4.5. Let $X$ be a $\mathbb{Q}$-factorial compact Kähler threefold with terminal singularities that is not uniruled. Then there exists a countable family $\left(\Gamma_{i}\right)_{i \in I}$ of rational curves on $X$ such that

$$
0<-K_{X} \cdot \Gamma_{i} \leq 4
$$

and

$$
\overline{N A}(X)=\overline{N A}(X)_{K_{X} \geq 0}+\sum_{i \in I} \mathbb{R}^{+}\left[\Gamma_{i}\right]
$$

Proof. As before assume that $X$ is smooth. The idea is to show that on the $K_{X}$-negative side, the cones $\overline{\mathrm{NE}}(X)$ and $\overline{\mathrm{NA}}(X)$ are quite similar, so the statement reduces to Proposition 4.4. More precisely we know by [DP04, Cor.0.3]2, that $\overline{\mathrm{NA}}(X)$ is the closure of the convex cone generated by cohomology classes of the form $[\omega]^{2},[\omega] \cdot[S]$ and $[C]$ where $\omega$ is a Kähler form, $S$ a surface and $C$ a curve on $X$. Note that [DP04, Cor.0.3] and [CT16 are general results, valid in any dimension.

Since $K_{X}$ is pseudoeffective we have $K_{X} \cdot[\omega]^{2} \geq 0$, so these classes lie in $\overline{\mathrm{NA}}(X)_{K_{X} \geq 0}$ and are not of any interest for the statement. The case of a curve class $[C]$ is dealt with by Proposition 4.4, so the main problem is to understand the classes $[\omega] \cdot[S]$ which are $K_{X}$-negative. Now observe that

$$
0>K_{X} \cdot[\omega] \cdot[S]=\left.K_{X}\right|_{S} \cdot\left[\left.\omega\right|_{S}\right] .
$$

Since $\left.\omega\right|_{S}$ is Kähler, the restriction $\left.K_{X}\right|_{S}$ is not pseudoeffective, hence the surface $S$ is covered by rational curves by Lemma 4.3. Yet this implies that (up to replacing $S$ by some resolution) that $H^{2}\left(S, \mathcal{O}_{S}\right)=0$, hence $S$ is projective and the cohomology class $\left[\left.\omega\right|_{S}\right]$ is an ample $\mathbb{R}$-divisor. Thus we can represent the class $[\omega] \cdot[S]$ by some

${ }^{2}$ If $X$ is not smooth, all the computations are on a resolution of singularities, cf. HP16, Sect.6]. We expect that DP04 Cor.0.3] holds for singular spaces with mild singularities, see CT16 for some progress in this direction. 
curve class $[C]$ where $C \subset S$ is an effective $\mathbb{R}$-divisor linearly equivalent to to the ample divisor $\left[\left.\omega\right|_{S}\right]$.

4.2. Contraction theorem - non-uniruled case. Let $X$ be a $\mathbb{Q}$-factorial compact Kähler threefold with terminal singularities that is not uniruled. We fix a $K_{X}$-negative extremal ray $R$ in the generalised Mori cone $\overline{\mathrm{NA}}(X)$. As a consequence of the cone theorem there exists a nef cohomology class $\alpha \in N^{1}(X)$ such that

$$
\alpha^{\perp} \cap \overline{\mathrm{NA}}(X):=\{\gamma \in \overline{\mathrm{NA}}(X) \mid \alpha \cdot \gamma=0\}=R
$$

and $\alpha$ is adjoint, i.e. we can write $\alpha=K_{X}+\omega$ with $\omega$ a Kähler class. If $X$ is a projective manifold one can choose $\alpha$ to be the class of a line bundle $L$ and the basepoint free theorem 2.3 tells us that some positive multiple of $L$ is generated by its global sections. The morphism defined by this multiple is then the contraction of the extremal ray $R$.

In the general setting of Kähler manifolds it is not clear whether $\alpha$ represents a line bundle, in fact a morphism between non-algebraic spaces is almost never defined by a line bundle. The only general tool for constructing morphisms in the analytic setting is given by the contraction theorems of Grauert, Fujiki and Ancona-van Tan:

Theorem 4.6. AT84 Let $X$ be a complex space, and let $Z \subset X$ be a closed complex subspace. Suppose that there exists a proper morphism $g: Z \rightarrow W$ such that the conormal sheaf $I_{Z} / I_{Z}^{2}$ of $Z \subset X$ is ample on the $g$-fibres. Suppose also that the natural map

$$
g_{*}\left(\mathcal{O}_{Z} / I_{Z}^{n+1}\right) \rightarrow g_{*}\left(\mathcal{O}_{Z} / I_{Z}^{n}\right)
$$

is surjective for any $n \geq 1$.

Then there exists a morphism $f: X \rightarrow Y$ such that $\left.f\right|_{X \backslash Z}$ is an isomorphism onto its image and $\left.f\right|_{Z}=g$.

In our setting the natural candidate for the subspace $Z$ is the locus covered by the curves in the extremal ray $R$. However from this description it is not clear how to check the conditions of Theorem 4.6. Moreover the theorem does not tell us how to check that $Y$ is a Kähler space. In order to overcome both of these difficulties we work with the cohomology class $\alpha$.

Let us note first that since $\alpha=K_{X}+\omega$ is the sum of a pseudoeffective class and a Kähler class, it is big, so we have $\alpha^{3}>0$. Thus the null locus

$$
\operatorname{Null}(\alpha):=\bigcup_{V \subset X, \alpha^{\operatorname{dim}} V \cdot V=0} V
$$

is a countable union of proper subvarieties of $X$. By a theorem of Collins-Tosatti CT15. (we argue on a resolution in order to be able to apply [CT15]), this locus is actually equal to the non-Kähler locus of $\alpha$. Modulo some technical arguments one can prove that $\operatorname{Null}(\alpha)$ is exactly the locus covered by curves in the extremal ray $R$ (in the algebraic case this follows a posteriori from the existence of the contraction). Since we are in dimension three we obtain the following cases

- $\operatorname{Null}(\alpha)$ is a finite union of curves;

- $\operatorname{Null}(\alpha)$ is an irreducible divisor $S$ and $\left.\alpha\right|_{S} \neq 0$;

- $\operatorname{Null}(\alpha)$ is an irreducible divisor $S$ and $\left.\alpha\right|_{S}=0$. 
In the first case we want to contract the curves via a small contraction onto points, in the second case we want to contract the divisor $S$ onto a curve, and in the last case we want to contract $S$ onto a point. We will deal with the first two cases, the last (and easiest) one is left to the interested reader.

Case 1: $\operatorname{Null}(\alpha)$ is a finite union of curves. In this case the morphism $g$ in Theorem 4.6 is simply the morphism that maps each connected component of $\operatorname{Null}(\alpha)$ onto a point. The difficult part is to check that the conormal sheaf is ample on each of the fibres. However we know from Bou04 that there exists a modification $\mu: X^{\prime} \rightarrow X$ such that the image of the exceptional locus is $\operatorname{Null}(\alpha)$ and

$$
\mu^{*} \alpha=\omega^{\prime}+E
$$

where $\omega^{\prime}$ is a Kähler class and $E$ an effective divisor. Since $\alpha$ is numerically trivial on every curve $C \subset \operatorname{Null}(\alpha)$, we obtain

$$
-\left.E\right|_{E}=\left.\omega^{\prime}\right|_{E}
$$

Thus the conormal sheaf of $E$ is ample and we can contract the connected components of $E$ onto points by applying Theorem 4.6 to $E \subset X^{\prime}$. By construction this induces the contraction on $X$.

Case 2: $\operatorname{Null}(\alpha)$ is an irreducible divisor $S$ and $\left.\alpha\right|_{S} \neq 0$. This case is surprisingly difficult. We can restate the conditions in a more geometrical way: the curves in the extremal ray $R$ cover the surface $S$, but it is not possible to connect two arbitrary points in $S$ using curves in $R$. Thus we expect that the curves in the extremal ray define a fibration $g: S \rightarrow C$ onto a curve $C$ and the extremal contraction will then contract the divisor $S$ onto this curve $C$. The problem is that in general the divisor $S$ can be non-normal, so standard techniques to define the fibration $g\left[\mathbf{B C E}^{+} \mathbf{0 2}\right.$ do not apply. One therefore defines first a fibration $\tilde{g}: \tilde{S} \rightarrow \tilde{C}$ on the normalisation $\tilde{S}$ of $S$. If $X$ is terminal (and some other cases, cf. CHP16. Ch.4.B]) a computation of intersection numbers shows that $S$ is smooth or at least slc in a neighbourhood of a general fibre. An explicit construction then shows that $\tilde{g}$ descends to a fibration $g$. Once we have defined the fibration $g$, the conditions on the conormal sheaf are easily verified since $S$ is a $\mathbb{Q}$-Cartier divisor.

We can now state the contraction theorem:

THEOREM 4.7. Let $X$ be a $\mathbb{Q}$-factorial compact Kähler threefold with terminal singularities that is not uniruled, and let $R \subset \overline{N A}(X)$ be a $K_{X}$-negative extremal ray. Then there exists a bimeromorphic morphism $\varphi: X \rightarrow Y$ onto a normal compact Kähler space such that $-K_{X}$ is $\varphi$-ample (so $\varphi$ is a projective morphism) and $\varphi$ contracts exactly the curves in the extremal ray $R$.

Proof. The considerations above establish the existence of a morphism $\varphi$ : $X \rightarrow Y$ in the category of compact analytic spaces. Thus we are left to show that $Y$ is also a Kähler space which can be done by checking the intersection properties in Lemma 3.10. We have already seen that $\alpha^{3}>0$ and by the definition of $\alpha$ we have $\alpha \cdot C=0$ for a curve $C$ if and only if $[C] \in R$. Hence the critical point is to show that $\alpha^{2} \cdot S=0$ if and only if $S$ is contracted by $\varphi$. Since $\alpha=K_{X}+\omega$ any such surface has the property that $\left.K_{X}\right|_{S}$ is not pseudoeffective, hence $S$ is covered by rational curves by Lemma 4.3. One proves [HP16, Prop.7.11] that $S$ is actually covered by $\alpha$-trivial rational curves, so $S$ is contracted by $\varphi$. 
4.3. Running the MMP - non-uniruled case. We are now in position to establish Theorem 1.2. So let $X$ be a normal $\mathbb{Q}$-factorial compact Kähler threefold with terminal singularities and assume that $X$ is not uniruled. If $K_{X}$ is nef, there is nothing to prove. If $K_{X}$ is not nef, then by Theorem 4.5, there exists a $K_{X^{-}}$ negative extremal ray $R \subset \overline{N A}(X)$. By Theorem 4.7 the contraction $\varphi: X \rightarrow Y$ exists. Since $X$ is not uniruled, $\varphi$ is birational. If $\varphi$ is divisorial, then $Y$ is again a normal $\mathbb{Q}$-factorial compact Kähler threefold with at most terminal singularities and we continue the MMP with $Y$ replacing $X$. If $\varphi$ is small, then by Mori's flip theorem Mor88, the flip: $X^{+} \rightarrow Y$ exists. Note here that the construction of the flip is analytically local around the exceptional locus of $\varphi$. Again, $X^{+}$is a normal $\mathbb{Q}$-factorial compact Kähler threefold with at most terminal singularities, and we may continue with $X^{+}$instead of $X$. Since the second Betti number drops by one in case of a divisorial contraction, it only remains to see that there is no infinite sequence of flips. This is however verified with exactly the same arguments as in the projective case, see KMM87.

4.4. Uniruled threefolds. Let $X$ be a $\mathbb{Q}$-factorial compact Kähler threefold with terminal singularities that is uniruled. Then the canonical class $K_{X}$ is not pseudoeffective, so it seems that the arguments from the preceding sections can not be used to establish the minimal model program. On the other hand the structure of non-algebraic uniruled Kähler threefolds is very simple: let $X \rightarrow Y$ be the MRC-fibration. If $Y$ had dimension at most one, then $H^{0}\left(X, \Omega_{X}^{2}\right)=0$, so $X$ is projective by Kodaira's criterion. Thus we see that $Y$ is a surface and the general fibre $F$ is isomorphic to $\mathbb{P}^{1}$. Choose now a Kähler class $\omega$ on $X$ such that $\left(K_{X}+\omega\right) \cdot F=0$. Then it follows from a result of Păun Pău12 that $K_{X}+\omega$ is pseudoeffective and we can develop the same theory as before, but now we only contract extremal rays that are $K_{X}+\omega$-negative. Running this MMP we obtain a bimeromorphic morphism $X \rightarrow X^{\prime}$ such that $K_{X^{\prime}}+\omega^{\prime}$ is nef. Such a class has very special properties:

TheOREM 4.8. Let $X$ be a normal $\mathbb{Q}$-factorial compact Kähler threefold with terminal singularities. Suppose that the base of the $M R C$-fibration $X \rightarrow Z$ has dimension two. Let $\omega$ be a Kähler class on $X$ such that $K_{X}+\omega$ is nef and $\left(K_{X}+\right.$ $\omega) \cdot F=0$ for $F$ a general fibre of the MRC-fibration.

Then there exists a holomorphic fibration $\varphi: X \rightarrow S$ onto a normal compact Kähler surface $S$ such that $K_{X}+\omega=\varphi^{*} \omega_{Y}$ where $\omega_{Y}$ is a Kähler class on $S$.

This statement is an analytic analogue of the basepoint free theorem: if $K_{X}+\omega$ is the class of a line bundle $L$, the statement says that $L$ is the pull-back of an ample divisor, in particular some multiple of $L$ is globally generated. The morphism $\varphi$ itself is in general not the contraction of an extremal ray, but $-K_{X}$ is $\varphi$-ample, so $\varphi$ is a projective morphism. By [Nak87. we can thus run a relative MMP over $S$ until we reach a Mori fibre space. Thus we may state

Corollary 4.9. Let $X$ be a normal non-algebraic uniruled $\mathbb{Q}$-factorial compact Kähler threefold with terminal singularities. Then $X$ is a bimeromorphic via a sequence of contractions of extremal rays in $\overline{N A}$ and of flips to a Mori fiber space $\varphi: X^{\prime} \rightarrow S$. Here $X^{\prime}$ is a normal $\mathbb{Q}$-factorial compact Kähler threefold with terminal singularities, $S$ is a normal non-algebraic Kähler surface, $-K_{X^{\prime}}$ is $\varphi$-ample and $b_{2}\left(X^{\prime}\right)=b_{2}(S)+1$. 
For the proof of Theorem 4.8 we use the nef reduction introduced in $\mathbf{B C E}^{+} \mathbf{0 2}$. This yields an almost holomorphic map $\varphi: X \rightarrow S$ onto some surface $S$ and the goal is to show that in our case this map is actually holomorphic. The key point of our argument is to show, via a second application of Păun's theorem [Pău12] that the numerical dimension of $K_{X}+\omega$ is two (hence equal to the "nef dimension" $\mathrm{BCE}^{+} \mathbf{0 2}$ ).

\section{Abundance for Kähler threefolds}

In Section 4 we explained that every Kähler threefold is bimeromorphic either to a Mori fibre space or a minimal model (i.e. a Kähler space with nef canonical class). In this section we will explain that such a minimal model is always good (i.e. the canonical class is semiample).

TheOrem 5.1. CHP16 Let $X$ be a normal $\mathbb{Q}$-factorial compact Kähler threefold with terminal singularities such that $K_{X}$ is nef. Then $K_{X}$ is semi-ample, that is some positive multiple $m K_{X}$ is globally generated.

As in the algebraic case, the proof of the abundance theorem falls into two parts:

TheOREM 5.2. DP03 Let $X$ be a normal $\mathbb{Q}$-factorial compact Kähler threefold with terminal singularities such that $K_{X}$ is nef. Then we have $\kappa(X) \geq 0$.

and

TheOREM 5.3. CHP16 Let $X$ be a normal $\mathbb{Q}$-factorial compact Kähler threefold with terminal singularities such that $K_{X}$ is nef. If $\kappa(X) \geq 0$, then $K_{X}$ is semi-ample.

5.1. Existence of a section. The existence of a global section for some pluricanonical divisor is a very difficult problem which, even for projective threefolds, is done by several case distinctions and ad-hoc arguments (cf. [LP16] for some recent progress). For non-algebraic Kähler threefolds, Theorem 5.2 was established in Pet01 with the exception that $X$ is simple (see Definition 6.1) and not bimeromorphic to a quotient of a torus. As a consequence of the abundance theorem 5.1 we will prove in Section 6 that this exceptional case does not exist, but a priori one has to develop tools that do not rely on this kind of classification result. In fact the paper Pet01] uses heavily the classification of non-algebraic Kähler threefolds which are not simple (due to Fujiki Fuj83), the remaining most difficult case was then settled in DP03. A key ingredient is the Kawamata-Viehweg type vanishing:

TheOREM 5.4. DP03 Let $X$ be a normal compact Kähler space of dimension $n$. Let $L$ be a nef line bundle on $X$ such that $c_{1}(L)^{2} \neq 0$. Then we have

$$
H^{q}\left(X, K_{X}+L\right)=0
$$

for $q \geq n-1$.

Actually, much more should be true:

CONJECTURE 5.5. Let $X$ be a normal compact Kähler with canonical singularities, and let $L$ be a nef line bundle on $X$ of numerical dimension $\nu(L)$ (see Definition 5.6). Then we have

$$
H^{q}\left(X, K_{X}+L\right)=0
$$

provided $q>n-\nu$. 
In case $X$ is projective, this is the (generalised) Kawamata-Viehweg theorem Kaw82 or Dem01, 6.13]. Notice that if $q=\operatorname{dim} X-1$, no assumption on the singularities is needed. Moreover, using the Grauert- Riemenschneider vanishing theorem

$$
R^{q} \pi_{*}\left(K_{\hat{X}}\right)=0
$$

for $q>0$ and any desingularisation $\pi: \hat{X} \rightarrow X$, it suffices to treat the smooth case. For new results towards Conjecture [5.5, we refer to Cao14 and to the recent solution of Demailly's strong openness conjecture GZ15.

The second main ingredient is the inequality

$$
K_{X} \cdot c_{2}(X) \geq 0
$$

for a minimal simply connected Kähler threefold $X$ with algebraic dimension $a(X)=$ 0 ; i.e. $X$ does not carry a non-constant meromorphic function. We will recover this inequality by different arguments in Section 5.4, here we explain the argument from DP03 which is of independent interest: philosophically speaking, this inequality comes from Enoki's theorem that the tangent sheaf of $X$ is $K_{X}$-semi-stable when $K_{X}^{2} \neq 0$ resp. $\left(K_{X}, \omega\right)$-semi-stable when $K_{X}^{2}=0$ (see Definition 5.11) where $\omega$ is any Kähler form on $X$. Now if this semi-stability with respect to a degenerate polarization would yield a Miyaoka-Yau inequality, then $K_{X} \cdot c_{2}(X) \geq 0$ would follow. However this type of Miyaoka-Yau inequalities with respect to degenerate polarizations is not known. In the projective case, the inequality is deduced from Miyaoka's generic nefness theorem (which uses char. $p$-methods). Instead the paper [DP03, approximates $K_{X}$ (in cohomology) by Kähler forms $\omega_{j}$. If $T_{X}$ is still $\omega_{j}$-semi-stable for sufficiently large $j$, then the usual Miyaoka-Yau inequality can be applied and in the limit the inequality $K_{X} \cdot c_{2}(X) \geq 0$ is established. Otherwise one examines the maximal destabilizing subsheaf which essentially (because of $a(X)=0)$ is independent of the polarization.

The third main ingredient is to prove the boundedness $h^{2}\left(X, m K_{X}\right) \leq 1$ in the case $\nu(X)=1$ (the case $\nu(X)=2$ is Theorem 5.4). This boundedness is shown under the additional assumption that $a(X)=0$ and that $\pi_{1}(X)$ is finite (otherwise by a result of Campana $X$ is already bimeromorphic to a quotient of a torus). The main point is that if $h^{2}\left(X, m K_{X}\right) \geq 2$, then we obtain "many" non-split extensions

$$
0 \rightarrow K_{X} \rightarrow \mathcal{E} \rightarrow m K_{X} \rightarrow 0
$$

and we analyze whether $\mathcal{E}$ is semi-stable or not. The assumption on $\pi_{1}$ is used to conclude that if $\mathcal{E}$ is projectively flat, then $\mathcal{E}$ is trivial after a finite étale cover.

From these three ingredients, Theorem 5.2 follows by applying Riemann-Roch calculations on a desingularisation of $X$.

5.2. Semi-ampleness: The general strategy. For the discussion of Theorem 5.3. we recall the definition of the numerical dimension:

Definition 5.6. Let $X$ be a normal compact Kähler space, and let $L$ be a nef line bundle on $X$. Then the numerical dimension of $L$ is given by

$$
\nu(X):=\max \left\{m \in \mathbb{N} \mid c_{1}(L)^{m} \not \equiv 0\right\} .
$$

If $K_{X}$ is $\mathbb{Q}$-Cartier of index $d$, we set

$$
\nu(X):=\nu\left(d K_{X}\right)
$$

for the numerical dimension of $X$. 
It is easy to see that

$$
\kappa(L) \leq \nu(L)
$$

and if $L$ is semi-ample, then equality holds. Kawamata Kaw85a discovered that if $L$ is the canonical class, then the converse holds:

THEOREM 5.7. Let $X$ be a normal compact Kähler space with only canonical singularities. Assume that $K_{X}$ is nef and that $\kappa(X)=\nu(X)$. Then $K_{X}$ is semiample.

The paper Kaw85a deals with the algebraic case, but the methods also work for Kähler spaces, see also [Nak87 and Fuj11.

If $\nu(X)=\operatorname{dim} X$, then $K_{X}$ is big. Since a Kähler Moishezon space with rational singularities is projective Nam02, we can apply the base point free theorem to see that $K_{X}$ is semiample. If $\nu(X)=0$, and if we assume $\kappa(X) \geq 0$, then $m K_{X}=\mathcal{O}_{X}$ for some positive number $m \in \mathbb{N}$. The potential case that $\kappa(X)=1$ and $\nu(X)=2$ is ruled out exactly as in the algebraic case, Kaw85b, 7.3]

Conclusion 5.8. In order to prove Theorem 5.3, it suffices to rule out the cases

$$
\kappa(X)=0, \nu(X)=1,2 .
$$

Since the Kodaira dimension is non-negative, there is a positive number $m \in \mathbb{N}$ and an effective divisor

$$
D \in\left|m K_{X}\right| \text {. }
$$

The standard method to prove that $\kappa(X) \geq 1$ is to consider the restriction map

$$
r: H^{0}\left(X, d(m+1) K_{X}\right) \rightarrow H^{0}\left(D, d K_{D}\right)
$$

for a suitable positive integer $d$. Arguing by induction on the dimension we aim to prove that $H^{0}\left(D, d K_{D}\right) \neq 0$ for some $d \in \mathbb{N}$ and that some non-zero section $u \in H^{0}\left(D, d K_{D}\right)$ lifts via $r$ to a global section $\tilde{u} \in H^{0}\left(X, d(m+1) K_{X}\right)$ on $X$. However, $D$ might be very singular and therefore it is not possible to analyse the divisor $D$ directly. In order to circumvent this difficulty, Kawamata Kaw92 developed the strategy, further explored in Kwc92, to consider log pairs $(X, B)$ with $B=\operatorname{Supp} D$ and to improve the singularities of this pair via certain birational transformations. This requires deep techniques of birational geometry of pairs within the theory of minimal models. In particular we have to run a log MMP for certain log pairs $(X, \Delta)$, which can be stated as follows.

\subsection{Semi-ampleness: Use of a log-MMP.}

TheOREM 5.9. CHP16 Let $X$ be a normal $\mathbb{Q}$-factorial compact Kähler threefold which is not uniruled. Let $D \in\left|m K_{X}\right|$ be a pluricanonical divisor and set $B:=\operatorname{Supp} D$. Suppose that the pair $(X, B)$ is dlt. Then there exists a terminating $(K+B)-M M P$, that is, there exists a bimeromorphic map

$$
\varphi:(X, B) \rightarrow\left(X^{\prime}, B^{\prime}:=\varphi_{*} B\right)
$$

which is a composition of $K+B$-negative divisorial contractions and flips such that $X^{\prime}$ is a normal $\mathbb{Q}$-factorial compact Kähler threefold, the pair $\left(X^{\prime}, B^{\prime}\right)$ is dlt and $K_{X^{\prime}}+B^{\prime}$ is nef. 
This is of course not the general log-MMP for Kähler threefolds, but it is sufficient for our purposes. The main obstacle for a general theorem is to show the existence of contractions for dlt pairs $(X, B)$ where $B$ has non-integer coefficients.

We will not explain the proof of this result [CHP16, Sect.3, Sect.4] here, it requires several improvements of the arguments we have seen in Section 4. Let us rather consider its consequences: we consider the most complicated case $\nu(X)=2$. Our goal is to prove that $\kappa(X) \geq 1$. Using the MMP of Theorem 5.9, this can be reduced by very technical arguments to the following:

Proposition 5.10. Let $X$ be a normal $\mathbb{Q}$-factorial compact Kähler threefold with at most klt singularities. Suppose that there exists a divisor $D \in\left|m K_{X}\right|$ with the following properties:

(1) Set $B:=\operatorname{Supp} D$. The pair $(X, B)$ is lc and $X \backslash B$ has terminal singularities.

(2) The divisor $K_{X}+B$ is nef and we have $\nu\left(K_{X}+B\right)=2$. Moreover we have $\kappa(X)=\kappa\left(K_{X}+B\right)$.

(3) For every irreducible component $T \subset B$ we have $\left.\left(K_{X}+B\right)\right|_{T} \neq 0$.

(4) We have $\left(K_{X}+B\right) \cdot K_{X}^{2} \geq 0$.

Then $\kappa(X) \geq 1$.

The acroynym klt stands for "Kawamata log terminal", and the definition is the same as in the algebraic setting. Properly speaking, $X$ is klt if there is a positive integer $m$ such that $\left(\omega_{X}^{\otimes m}\right)^{* *}$ is locally free and there is a resolution $\pi: \tilde{X} \rightarrow X$ such that

$$
\omega_{\tilde{X}}^{\otimes m}=\pi^{*}\left(\left(\omega_{X}^{\otimes m}\right)^{* *}\right) \otimes \mathcal{O}_{\tilde{X}}\left(\sum a_{i} m E_{i}\right),
$$

where the $E_{i}$ are the $\pi$-exceptional divisors and all $a_{i}>-1$. In other words, we have the usual equation

$$
K_{\tilde{X}}=\pi^{*}\left(K_{X}\right)+\sum a_{i} E_{i}
$$

with $a_{i}>-1$.

We will explain the proof of this proposition in Subsection 5.5, but this requires another ingredient:

5.4. Semi-ampleness: Generic nefness and Chern class inequalities. We saw in Subsection 5.1 that a Chern class inequality in the spirit of Miy87, 6.1] plays a crucial role for nonvanishing, this problem appears again for abundance. Having in mind our application, we restrict ourselves to threefolds; moreover we shall need - and this is crucial - only to consider threefolds with isolated singularities. We do not make any attempt to define Chern classes of coherent sheaves on singular spaces, all that we need are the intersection numbers

$$
\alpha \cdot c_{1}(\mathcal{F})^{2}, \alpha \cdot c_{2}(\mathcal{F}), \alpha^{2} \cdot c_{1}(\mathcal{F}) .
$$

where $\alpha \in N^{1}(X)$. For example, let us define $\alpha \cdot c_{2}(\mathcal{F})$. Choose a desingularisation $\pi: \hat{X} \rightarrow X$; then $c_{2}\left(\pi^{*}(\mathcal{F})\right) \in H^{4}(\hat{X}, \mathbb{Z})$ is well-defined, and we set

$$
\alpha \cdot c_{2}(\mathcal{F})=\pi^{*}(\alpha) \cdot c_{2}\left(\pi^{*}(\mathcal{F})\right) .
$$

See [CHP16, 7.1] for details. In particular, slope stability with respect to a nef class can be defined: 
Definition 5.11. Let $X$ be a normal compact Kähler threefold with isolated singularities and let $\alpha$ be a nef class on $X$. We say that a non-zero torsion-free sheaf $\mathcal{F}$ is $\alpha$-semistable (resp. $\alpha$-stable) if for every non-zero saturated subsheaf $\mathcal{E} \subset \mathcal{F}$ we have

$$
\mu_{\alpha}(\mathcal{E}):=\frac{\alpha^{2} \cdot c_{1}(\mathcal{E})}{\operatorname{rk} \mathcal{E}} \leq \frac{\alpha^{2} \cdot c_{1}(\mathcal{F})}{\operatorname{rk} \mathcal{F}}=: \mu_{\alpha}(\mathcal{F}) \quad(\text { resp. }<) .
$$

The generic nefness notion we shall use is given in

Definition 5.12. Let $X$ be a normal compact Kähler threefold, and let $\alpha$ be a nef class on $X$. A non-zero torsion-free coherent sheaf $\mathcal{F}$ on $X$ is $\alpha$-generically nef if for every torsion-free quotient sheaf $\mathcal{F} \rightarrow Q \rightarrow 0$ we have

$$
\alpha^{2} \cdot c_{1}(Q) \geq 0 .
$$

In this setting the following Chern class inequality holds.

THEOREM 5.13. Let $(X, \omega)$ be a compact Kähler threefold with isolated singularities. Let $\mathcal{F}$ be a non-zero reflexive coherent sheaf on $X$ such that $\operatorname{det} \mathcal{F}$ is $\mathbb{Q}$-Cartier. Suppose that there exists a pseudoeffective class $P \in N^{1}(X)$ such that

$$
L:=c_{1}(\mathcal{F})+P
$$

is a nef class. Suppose furthermore that for all $0<\varepsilon \ll 1$ the sheaf $\mathcal{F}$ is $(L+\varepsilon \omega)$ generically nef. Then we have

$$
L \cdot c_{2}(\mathcal{F}) \geq \frac{1}{2}\left(L \cdot c_{1}^{2}(\mathcal{F})-L^{3}\right) .
$$

In particular, if $L \cdot c_{1}^{2}(\mathcal{F}) \geq 0$ and $L^{3}=0$, then

$$
L \cdot c_{2}(\mathcal{F}) \geq 0 .
$$

The proof is based on a Bogomolov inequality for stable sheaves on Kähler threefolds with isolated singularities which we state next.

TheOREM 5.14. Let $X$ be a normal compact Kähler threefold with isolated singularities, and let $\alpha$ be a Kähler class on $X$. Let $\mathcal{F}$ be an $\alpha$-stable non-zero torsion-free coherent sheaf on $X$. Then we have

$$
\alpha \cdot c_{2}(\mathcal{F}) \geq\left(\frac{r-1}{2 r}\right) \alpha \cdot c_{1}^{2}(\mathcal{F}) .
$$

Theorem 5.13 can be applied to $\left(\Omega_{X}\right)^{* *}$ (or $\Omega_{X} /$ torsion) since we have the following

Proposition 5.15. Let $X$ be a normal compact Kähler space of dimension $n$ with canonical singularities. Suppose that $K_{X}$ is nef or $\kappa(X) \geq 0$. Then $\Omega_{X} /$ torsion is generically nef with respect to any nef class $\alpha$, i.e. for every torsion-free quotient sheaf

$$
\Omega_{X} \rightarrow \mathcal{Q} \rightarrow 0
$$

we have $\alpha^{n-1} \cdot c_{1}(\mathcal{Q}) \geq 0$.

This result is a Kähler version of Miyaoka's generic nefness theorem which makes the weaker assumption that $K_{X}$ is pseudoeffective. Our proof is by reduction to Enoki's theorem Eno88, which makes use of the solution of a Monge-Ampère equation. 
5.5. Semi-ampleness: proof of Proposition 5.10, Running some relative MMP one sees that there exists a terminal modification

$$
\mu: X^{\prime} \rightarrow X
$$

of $X$, i.e., $X^{\prime}$ has only terminal singularities, and there exists an effective $\mathbb{Q}$-divisor $\Delta$ such that

$$
K_{X^{\prime}}+\Delta \sim_{\mathbb{Q}} \mu^{*} K_{X} .
$$

Choose $m>0$ such that $m\left(K_{X}+B\right)=: L$ is Cartier and set $L^{\prime}:=\mu^{*}(L)$. The key is now the basic Chern class inequality

$$
L^{\prime} \cdot\left(K_{X^{\prime}}^{2}+c_{2}\left(X^{\prime}\right)\right) \geq 0
$$

which comes down to proving that $L^{\prime} \cdot c_{2}\left(X^{\prime}\right) \geq 0$. Now by Proposition 5.15, the sheaf $\Omega_{X^{\prime}}$ is generically nef. Since

$$
K_{X^{\prime}}+\Delta+\mu^{*} B \sim_{\mathbb{Q}} \mu^{*}\left(K_{X}+B\right)
$$

is nef, the conditions of Theorem 5.13 are satisfied with $\mathcal{F}:=\left(\Omega_{X^{\prime}}\right)^{* *}$ and $P:=$ $\Delta+\mu^{*} B$. Thus we conclude

$$
L^{\prime} \cdot c_{2}\left(X^{\prime}\right) \geq 0,
$$

hence the Chern class inequality (5.2) is established. Now by a Riemann-Roch formula,

$$
\chi\left(X^{\prime}, \mathcal{O}_{X^{\prime}}\left(n L^{\prime}\right)\right)=n L^{\prime} \cdot \frac{K_{X^{\prime}}^{2}+c_{2}\left(X^{\prime}\right)}{12}+\chi\left(X^{\prime}, \mathcal{O}_{X^{\prime}}\right)
$$

for all $n \in \mathbb{N}$. Thus (5.2) yields a constant $k$ such that

$$
\chi\left(X^{\prime}, \mathcal{O}_{X^{\prime}}\left(n L^{\prime}\right)\right) \geq k
$$

for all $n \in \mathbb{N}$, and therefore

$$
\chi\left(X, \mathcal{O}_{X}(n L)\right) \geq k
$$

for all $n \in \mathbb{N}$. Now we show that $H^{3}\left(X, \mathcal{O}_{X}(n L)\right)=0$ and that $h^{2}\left(X, \mathcal{O}_{X}(n L)\right)$ is constant for large $n$, hence

$$
h^{0}(X, n L) \geq h^{1}(X, n L)+c
$$

with some constant $c \in \mathbb{Z}$. Now comes the inductive argument we have been aiming for: $B$ is a minimal surface with at most slc singularities and $\nu(B)=1$, so we know by abundance that $h^{0}\left(B, \mathcal{O}_{B}(n L)\right)$ grows linearly. The Euler characteristic $\chi\left(B, \mathcal{O}_{B}(n L)\right)$ is constant, moreover one can show

$$
h^{2}\left(B, \mathcal{O}_{B}(n L)\right)=0
$$

for $n \gg 0$. Thus $h^{1}\left(B, \mathcal{O}_{B}(n L)\right)$ also grows linearly. Using the restriction sequence (cf. [CHP16, Sect.8] for details) one deduces that $h^{1}\left(X, \mathcal{O}_{X}(n L)\right)$ grows at least linearly. Therefore we have

$$
\kappa(X)=\kappa\left(K_{X}+B\right)=\kappa(L) \geq 1 .
$$




\section{Applications}

6.1. Simple Kähler spaces. One of the initial motivations for the study of the Kähler MMP was to describe the "most non-algebraic" Kähler spaces, i.e. those that contain only a few subvarieties.

Definition 6.1. Let $X$ be a normal compact Kähler space. We say that $X$ is simple, if there is no proper positive-dimensional subvariety through a very general point of $X$.

Examples of simple Kähler spaces are general tori or general hyperkähler manifolds (say the Hilbert scheme $S^{[n]}$ of a K3 surface without curves). It is expected that all simple spaces arise from these two types of manifolds by standard constructions, i.e bimeromorphic transformations and finite maps. The MMP for Kähler threefolds confirms this in dimension three:

TheOREm 6.2. Let $X$ be a normal $\mathbb{Q}$-factorial compact Kähler threefold with terminal singularities. Suppose that $X$ is simple. Then there exists a bimeromorphic morphism $X \rightarrow T / G$ where $T$ is a torus and $G$ a finite group acting on $T$.

Proof. Since $X$ is not uniruled, we know by Theorem 1.2 that there exists a minimal model $X \rightarrow X^{\prime}$. Since $X$ (and hence $X^{\prime}$ ) is not covered by divisors we see that $\kappa\left(X^{\prime}\right)=0$. Since $K_{X^{\prime}}$ is semiample by Theorem 1.2 we have $\nu\left(X^{\prime}\right)=\kappa\left(X^{\prime}\right)=$ 0 , i.e. the canonical divisor $K_{X^{\prime}}$ is numerically trivial. Since $X$ (and hence $X^{\prime}$ ) is not covered by curves, the structure theorem 6.3, stated below, yields that

$$
X^{\prime} \simeq T / G
$$

with $T$ a torus and $G$ a finite group. Since $X$ (and hence $X^{\prime}$ ) is not covered by positive-dimensional subvarieties, the torus $T$ has no positive-dimensional subvarieties. In particular $T / G$ has no positive-dimensional subvarieties, so $X \rightarrow T / G$ extends to a morphism.

TheOREM 6.3. Let $X$ be a non-algebraic $\mathbb{Q}$-factorial compact Kähler threefold with terminal singularities. If $K_{X} \equiv 0$, there exists a Galois cover $f: \tilde{X} \rightarrow X$ that is étale in codimension one such that either $X$ is a torus or a product of an elliptic curve and a K3 surface.

This result should be understood as a generalisation of the Beauville-Bogomolov decomposition to singular Kähler threefolds, the proof is based on the observation that after a cyclic covering one has $\chi\left(X, \mathcal{O}_{X}\right)=0$ (cf. CHP16 for details and further structure results for non-algebraic Kähler threefolds). We refer to Cam04, GKP11, Dru16 for further information concerning the Beauville-Bogomolov decomposition.

\subsection{Algebraic approximation of Kähler spaces.}

Definition 6.4. Let $X$ be a normal compact Kähler space. We say that $X$ is algebraically approximable if there exists a proper flat morphism $\pi: \mathcal{X} \rightarrow B$ to a complex space $B$, such that the following holds.

(1) there is a point $0 \in B$ such that $X_{0} \simeq X$;

(2) there is a sequence $\left(t_{k}\right)_{k \in \mathbb{N}}$ in $B$, converging to 0 such that the fibre $X_{t_{k}}$ is a projective variety for all $k \in \mathbb{N}$. 
Originally, Kodaira asked whether any compact Kähler manifold can be approximated algebraically. However, C. Voisin constructed counterexamples in dimension at least 4 [Voi04]. In dimension at least 10, more is true: there are compact Kähler manifolds $X$ such that no smooth bimeromorphic model can be approximated algebraically Voi06. In view of the MMP, it is natural to consider minimal models of $X$, which are usually singular. So one can formulate:

Conjecture 6.5. (Modified Kodaira Conjecture)

Let $X$ be a compact Kähler manifold. Then there exists a bimeromorphic model $X \rightarrow X^{\prime}$ where $X^{\prime}$ is normal $\mathbb{Q}$-factorial compact Kähler space with terminal singularities that is algebraically approximable.

In fact if $X$ is not uniruled some minimal model of $X$ should be algebraically approximable. A first partial positive answer in dimension 3 is given by P.Graf:

Theorem 6.6. Gra16 Let $X$ be a smooth compact Kähler threefold with $\kappa(X)=0$. Then $X$ has a minimal model which is algebraically approximable.

Actually, the deformation Graf constructs is even locally trivial (in the sense that it preserves the singularities Gra16, Defn.2.10]).

\section{Outlook}

In this final section we would like to explain some of the challenges that will appear in the construction of the minimal model program for Kähler spaces of arbitrary dimension.

1. Existence of rational curves. In Subsection 4.1 we saw that the construction of rational curves for (not necessarily algebraic) Kähler threefolds is done in two steps: first Brunella's theorem on rank one foliations describes the case where the canonical class is not pseudo-effective, then the deformation theory of curves on threefolds allows to describe $K_{X}$-negative curves when $K_{X}$ is pseudoeffective. In higher dimension it is possible to replace the deformation theoretic part by a subadjunction argument CH15 that reduces the problem to the characterisation of uniruledness in lower dimension. More precisely the existence of rational curves for Kähler manifolds is reduced to the following difficult

Conjecture 7.1. Let $X$ be a compact Kähler manifold. Then the canonical class $K_{X}$ is pseudoeffective if and only if $X$ is not uniruled.

Of course, if $X$ is uniruled, then $K_{X}$ cannot be pseudo-effective. In case $X$ is projective, the conjecture is a theorem of Miyaoka-Mori, [MM86]; again the construction relies on char $p$ methods. Here completely new methods seem to be necessary. One step could be to establish the Kähler version of the main result in BDPP13.

2. Contracting extremal rays. The nef supporting class $\alpha$ introduced in Subsection 4.2 is an extremely useful tool that mimics the role of the line bundle $L$ appearing in the basepoint free theorem 2.3. However all the considerations aim at verifying the conditions of the Grauert-Fujiki-Ancona-Van Tan contraction theorem 4.6 which can be quite lengthy. In particular exhibiting the proper map on the exceptional locus/Null locus becomes even more difficult since even a posteriori exceptional loci of Mori contractions can be very singular. It seems that new ideas are necessary to deal with this problems, a good start would be a new version of Grauert's theorem with conditions that are more adapted to the Mori setting. 
3. Checking the Kähler property. One of the surprising features of the MMP for Kähler threefolds is that a posteriori the positivity properties of all the divisors (and cohomology classes) appearing in our proofs can be checked by intersecting with curves. The following example shows that already in dimension 4 this is no longer true:

EXAMPLE 7.2. Let $B$ be a K3 surface that does not contain any curves (in particular $B$ is Kähler, but not projective). Denote by $X^{\prime}$ the projectivisation of the rank three vector bundle $\Omega_{B} \oplus \mathcal{O}_{B}$, then $\psi: X^{\prime} \rightarrow B$ is a $\mathbb{P}^{2}$-bundle so $X^{\prime}$ is a compact Kähler fourfold. Denote by $S^{\prime} \subset X^{\prime}$ the $\psi$-section corresponding to the quotient line bundle $\Omega_{B} \oplus \mathcal{O}_{B} \rightarrow \mathcal{O}_{B}$, and let $\mu: X \rightarrow X^{\prime}$ be the blow-up of $X^{\prime}$ along the surface $S^{\prime}$. Then $X$ is a compact Kähler fourfold, and $\mu$ is the contraction of a $K_{X}$-negative extremal ray in $\overline{\mathrm{NA}}(X)$ (in particular $-K_{X}$ is relatively ample). Note that there does not exist a surface $S \subset X$ that dominates $S^{\prime} \subset X$ : indeed the $\mu$-exceptional divisor $E \rightarrow S^{\prime}$ is isomorphic to $\mathbb{P}\left(\Omega_{B}\right) \rightarrow B$ and it is easy to check that the $\mathbb{P}^{1}$-bundle $\mathbb{P}\left(\Omega_{B}\right) \rightarrow B$ does not admit any meromorphic multisections.

Challenge: let $\alpha$ be a nef supporting class (cf. Subsection 4.2) of the extremal ray contracted by $\mu$. Show that we can choose $\alpha$ such that $\alpha=\mu^{*} \omega$ with $\omega$ a Kähler class on $X^{\prime}$.

\section{References}

AT84. V. Ancona and Vo Van Tan. On the blowing down problem in C-analytic geometry. $J$. Reine Angew. Math., 350:178-182, 1984.

$\mathrm{BCE}^{+}$02. Thomas Bauer, Frédéric Campana, Thomas Eckl, Stefan Kebekus, Thomas Peternell, Sławomir Rams, Tomasz Szemberg, and Lorenz Wotzlaw. A reduction map for nef line bundles. In Complex geometry (Göttingen, 2000), pages 27-36. Springer, Berlin, 2002.

BCHM10. Caucher Birkar, Paolo Cascini, Christopher D. Hacon, and James McKernan. Existence of minimal models for varieties of log general type. J. Amer. Math. Soc., 23(2):405-468, 2010.

BDPP13. Sébastien Boucksom, Jean-Pierre Demailly, Mihai Păun, and Thomas Peternell. The pseudo-effective cone of a compact Kähler manifold and varieties of negative Kodaira dimension. Journal of Algebraic Geometry, 22:201-248, 2013.

Bou04. Sébastien Boucksom. Divisorial Zariski decompositions on compact complex manifolds. Ann. Sci. École Norm. Sup. (4), 37(1):45-76, 2004.

BPEG13. Sébastien Boucksom; Philippe Eyssidieux and Vincent Guedj, editors. An introduction to the Kähler-Ricci flow. Selected papers based on the presentations at several meetings of the ANR project MACK. Cham: Springer, 2013.

Bru06. Marco Brunella. A positivity property for foliations on compact Kähler manifolds. Internat. J. Math., 17(1):35-43, 2006.

Cam04. Frédéric Campana. Orbifoldes à première classe de Chern nulle. In The Fano Conference, pages 339-351. Univ. Torino, Turin, 2004.

Cao14. Junyan Cao. Numerical dimension and a Kawamata-Viehweg-Nadel-type vanishing theorem on compact Kähler manifolds. Compos. Math., 150(11):1869-1902, 2014.

CH15. Junyan Cao and Andreas Höring. Rational curves on compact Kähler manifolds. arXiv preprint, 1502.03936, 2015.

CHP16. Frédéric Campana, Andreas Höring, and Thomas Peternell. Abundance for Kähler threefolds. Annales de l'ENS, 49:969-1023, 2016.

CT15. Tristan C. Collins and Valentino Tosatti. Kähler currents and null loci. Invent. Math., 202(3):1167-1198, 2015.

CT16. Tristan C. Collins and Valentino Tosatti. A singular Demailly-Păun theorem. C. R. Math. Acad. Sci. Paris, 354(1):91-95, 2016.

Dem85. Jean-Pierre Demailly. Mesures de Monge-Ampère et caractérisation géométrique des variétés algébriques affines. Mém. Soc. Math. France (N.S.), (19):124, 1985. 
Dem92. Jean-Pierre Demailly. Regularization of closed positive currents and intersection theory. J. Algebraic Geom., 1(3):361-409, 1992.

Dem01. Jean-Pierre Demailly. Multiplier ideal sheaves and analytic methods in algebraic geometry. In School on Vanishing Theorems and Effective Results in Algebraic Geometry (Trieste, 2000), volume 6 of ICTP Lect. Notes, pages 1-148. Abdus Salam Int. Cent. Theoret. Phys., Trieste, 2001.

DP03. Jean-Pierre Demailly and Thomas Peternell. A Kawamata-Viehweg vanishing theorem on compact Kähler manifolds. J. Differential Geom., 63(2):231-277, 2003.

DP04. Jean-Pierre Demailly and Mihai Păun. Numerical characterization of the Kähler cone of a compact Kähler manifold. Ann. of Math. (2), 159(3):1247-1274, 2004.

Dru16. Stéphane Druel. A decomposition theorem for singular spaces with trivial canonical class of dimension at most five. arXiv preprint, 1606.09006, 2016.

Eno88. Ichiro Enoki. Stability and negativity for tangent sheaves of minimal Kähler spaces. In Geometry and analysis on manifolds (Katata/Kyoto, 1987), volume 1339 of Lecture Notes in Math., pages 118-126. Springer, Berlin, 1988.

Fuj83. Akira Fujiki. On the structure of compact complex manifolds in C. In Algebraic varieties and analytic varieties (Tokyo, 1981), volume 1 of Adv. Stud. Pure Math., pages 231-302. North-Holland, Amsterdam, 1983.

Fuj04. Osamu Fujino. Termination of 4-fold canonical flips. Publ. Res. Inst. Math. Sci., 40(1):231-237, 2004.

Fuj05. O. Fujino. Addendum to: "Termination of 4-fold canonical flips". Publ. Res. Inst. Math. Sci., 41(1):251-257, 2005.

Fuj11. Osamu Fujino. On Kawamata's theorem. In Classification of algebraic varieties, EMS Ser. Congr. Rep., pages 305-315. Eur. Math. Soc., Zürich, 2011.

GKP11. Daniel Greb, Stefan Kebekus, and Thomas Peternell. Singular spaces with trivial canonical class. arXiv preprint, to appear in Minimal models and extremal rays, Proceedings of the conference in honor of Shigefumi Mori's 60th birthday, Advanced Studies in Pure Mathematics, 1110.5250, 2011.

Gra62. Hans Grauert. Über Modifikationen und exzeptionelle analytische Mengen. Math. Ann., 146:331-368, 1962.

Gra16. Patrick Graf. Algebraic approximation of Kähler threefolds of Kodaira dimension zero. arXiv preprint, 1601.04307, 2016.

GZ15. Qi'an Guan and Xiangyu Zhou. A proof of Demailly's strong openness conjecture. Ann. of Math. (2), 182(2):605-616, 2015.

HP15. Andreas Höring and Thomas Peternell. Mori fibre spaces for Kähler threefolds. J. Math. Sci. Univ. Tokyo, 22(1):219-246, 2015.

HP16. Andreas Höring and Thomas Peternell. Minimal models for Kähler threefolds. Invent. Math., 203(1):217-264, 2016.

Kaw82. Yujiro Kawamata. Kodaira dimension of algebraic fiber spaces over curves. Invent. Math., 66(1):57-71, 1982.

Kaw85a. Y. Kawamata. Pluricanonical systems on minimal algebraic varieties. Invent. Math., 79(3):567-588, 1985.

Kaw85b. Yujiro Kawamata. Minimal models and the Kodaira dimension of algebraic fiber spaces. J. Reine Angew. Math., 363:1-46, 1985.

Kaw92. Yujiro Kawamata. Abundance theorem for minimal threefolds. Invent. Math., 108(2):229-246, 1992.

KM98. János Kollár and Shigefumi Mori. Birational geometry of algebraic varieties, volume 134 of Cambridge Tracts in Mathematics. Cambridge University Press, Cambridge, 1998. With the collaboration of C. H. Clemens and A. Corti.

KMM87. Yujiro Kawamata, Katsumi Matsuda, and Kenji Matsuki. Introduction to the minimal model problem. In Algebraic geometry, Sendai, 1985, volume 10 of Adv. Stud. Pure Math., pages 283-360. North-Holland, Amsterdam, 1987.

Kol96. János Kollár. Rational curves on algebraic varieties, volume 32 of Ergebnisse der Mathematik und ihrer Grenzgebiete. 3. Folge. A Series of Modern Surveys in Mathematics. Springer-Verlag, Berlin, 1996.

Kwc92. János Kollár (with 14 coauthors). Flips and abundance for algebraic threefolds. Société Mathématique de France, Paris, 1992. Papers from the Second Summer Seminar on 
Algebraic Geometry held at the University of Utah, Salt Lake City, Utah, August 1991, Astérisque No. 211 (1992).

LP16. Vladimir Lazić and Thomas Peternell. Abundance for varieties with many differential forms. arXiv preprint, 1601.01602, 2016.

Miy87. Yoichi Miyaoka. The Chern classes and Kodaira dimension of a minimal variety. In Algebraic geometry, Sendai, 1985, volume 10 of Adv. Stud. Pure Math., pages 449476. North-Holland, Amsterdam, 1987.

MM86. Yoichi Miyaoka and Shigefumi Mori. A numerical criterion for uniruledness. Ann. of Math. (2), 124(1):65-69, 1986.

Mor79. Shigefumi Mori. Projective manifolds with ample tangent bundles. Ann. of Math. (2), 110(3):593-606, 1979.

Mor82. Shigefumi Mori. Threefolds whose canonical bundles are not numerically effective. Ann. of Math. (2), 116(1):133-176, 1982.

Mor88. Shigefumi Mori. Flip theorem and the existence of minimal models for 3-folds. J. Amer. Math. Soc., 1(1):117-253, 1988.

Nak87. Noboru Nakayama. The lower semicontinuity of the plurigenera of complex varieties. In Algebraic geometry, Sendai, 1985, volume 10 of Adv. Stud. Pure Math., pages 551-590. North-Holland, Amsterdam, 1987.

Nam02. Yoshinori Namikawa. Projectivity criterion of Moishezon spaces and density of projective symplectic varieties. Internat. J. Math., 13(2):125-135, 2002.

Pău12. Mihai Păun. Relative adjoint transcendental classes and albanese maps of compact kaehler manifolds with nef ricci curvature. arXiv preprint, 1209.2195, 2012.

Pet01. Thomas Peternell. Towards a Mori theory on compact Kähler threefolds. III. Bull. Soc. Math. France, 129(3):339-356, 2001.

Sho92. V.V. Shokurov. 3-fold log flips. Appendix by Yujiro Kawamata: The minimal discrepancy coefficients of terminal singularities in dimension three. Russ. Acad. Sci., Izv., Math., 40(1):95-202, 1992.

Voi04. Claire Voisin. On the homotopy types of compact Kähler and complex projective manifolds. Invent. Math., 157(2):329-343, 2004.

Voi06. Claire Voisin. On integral Hodge classes on uniruled or Calabi-Yau threefolds. Mukai, Shigeru (ed.) et al., Moduli spaces and arithmetic geometry. Mathematical Society of Japan. Advanced Studies in Pure Mathematics 45, 43-73, 2006.

Andreas Höring, Université Côte d'Azur, CNRS, LJAD, France

E-mail address: hoering@unice.fr

Thomas Peternell, Mathematisches Institut, Universität Bayreuth, 95440 Bayreuth, GERMANy

E-mail address: thomas.peternell@uni-bayreuth.de 